\title{
The Effects of Craniosacral Techniques on the Kinematics of the Craniomandibular Joint - Kinematographic Validation
}

\author{
Johann Lechner ${ }^{1 *}$, Christian Langer ${ }^{2}$ and Nicholas J Meyer ${ }^{3}$ \\ ${ }^{1}$ Head of Clinic for Integrative Dentistry, Gruenwalder, Munich, Germany \\ ${ }^{2}$ Department of Dentistry, Würenlos, Switzerland \\ ${ }^{3}$ Department of Dentistry, USA \\ *Corresponding Author: Johann Lechner, Head of Clinic for Integrative Dentistry, \\ Gruenwalder, Munich, Germany.
}

DOI: $10.31080 /$ ASDS.2020.04.0784
Received: January 18, 2020

Published: February 13, 2020

(C) All rights are reserved by Johann

Lechner., et al.

\begin{abstract}
Introduction: There is considerable controversy surrounding craniosacral therapy (CsTh). To date, there have been few research studies examining the effects of CsTh when used to treat dysfunction of the musculature of the craniomandibular joint.

Materials and Methods: Five craniosacral techniques (CsT) were used and their effects recorded with time/motion analysis. The study was carried out under placebo-controlled conditions with the intention of providing relevant data. Opening and closing speeds (velocity) of the mandible were measured twice on all test subjects using the K7 measuring system (Myotronics, Tukwila, WA, USA) in order to objectively record and display graphically the kinetic differences pre- and post-CsTh treatment.

Results: The jaw closing speed was clearly increased after the initial CsTh treatment in both test subjects requiring treatment (dysfunctional group) and healthy test subjects. Terminal velocity at the time of tooth contact during rapid closing of the jaw was also significantly increased in both groups.

Conclusion: The results of this study demonstrate that where there exist craniomandibular dysfunctions that are not the result of problems with occlusion, the movement of the mandible could be influenced by CsTh treatment. This research indicates that the arthrokinetic dysfunctions in the musculature of the craniomandibular system may be positively influenced by CsT.
\end{abstract}

Keywords: Craniosacral Therapy; Craniomandibular Dysfunction; Kinematographic Validation

\section{Abbreviations}

CSTH: Craniosacral Therapy; CST: Craniosacral Techniques; CST: Craniosacral Therapist; TT: Terminal Tooth Contact; TT SL: Terminal Tooth Slow Contact; TT F: Terminal Tooth Fast Contact; OP SL: Opening Speed Slow; OP F: Opening Speed Fast; CL SL: Closing Speed Slow; CL F: Closing Speed Fast; PRE-CST: Pre-Cst Treatment; PRE-PL: Pre-Placebo/Sham Treatment; POST-CST: Post-Cst Treatment; POST-PL: Post Placebo/Sham Treatment

\section{Introduction}

Osteopathy in the cranial area, also referred to as "Osteopathy of the Cranial Field" (OCF), may be traced back to W.G. Sutherland, DO, who developed the concept in the 1940's. Craniosacral therapy
(CsTh) was developed from a particular form of osteopathy and, in its current form, was primarily shaped by osteopaths Upledger and Sutherland [1,2]. Many TMJ problems are caused by compression of the cervical joints, which involves dental and physical therapists to intercept many serious conditions. Following a coordinated approach to treatment is required [3]. Other authors emphasize the complexity of the joint system which is reflected in the range and diversity of the thorough examination [3]. Multimode treatment is necessary in the long-term resolution of the disorders and these range from medicinal, nutritional and psychological therapies to occlusal equilibration and physiotherapy [4]. There is agreement in literature that an association between the postural inclination of the cervical spine and the position of the mandible can 
be demonstrated [5]. Numerous studies have demonstrated functional interactions of a predominantly morphologic and neuromuscular nature which have been suspected to influence the entire system of cranial, cervical, dorsal and sacral structures: Any disturbance of one segment would affect the entire system [6]. Experiments have shown that changes of the occlusal height and jaw position led to changes of the upper cervical spine and evoked reactions of the motor and autonomic nervous system [7]. King was one of the first to measure the cranial rhythmic impulse by laser-Doppler flowmeter, which could in turn lead to definitive, evidence-based research $[8,9]$. To date, there has been no formal verification of the effectiveness of this treatment in the subarea of craniosacral osteopathy on craniosacral bone/"the craniosacral region" and there is a lack of objective validation in this field still totally $[10,11]$. Despite extensive literature on CsTh there have been limited research studies or documentation concerning the effects of CsT on craniomandibular dysfunctions [12-16]. Consequently there is only little data available to prove the efficacy of osteopathic and cranial manipulative treatment.

\section{Objectives}

The here presented paper tries an objective measurement of craniofacial techniques and aims to address the following questions: Are CsT effective, and if so, is it possible to measure the effect? Does OCF affect the kinematics of the craniomandibular complex? Is the kinesiographic measuring system, (i.e., jaw motion/ speed), suitable for verifying the effects of CsT with objective data?

\section{Material and Method}

A kinematic examination was performed on each test subject by a craniosacral therapist (CST) in collaboration with a dental specialist, enabling those test subjects requiring treatment to be differentiated from those who were healthy, and also identifying those subjects with occlusion difficulties. The K7 measuring system (Myotronics, Tukwila, WA, USA) was used to measure the extent to which each subject's jaw mechanics were affected by the techniques used during treatment. The K7 Evaluation System can aid measure and record the functioning of the mandibular and its associated masticatory muscle. The system monitors and records distinct sets of data which can aid evaluate the neuromuscular condition of the temporomandibular joint and assess its condition prior to and following treatment. The data capable of being measured includes electromyography and jaw tracking. The equipment has been specifically designed so that it does not get in the way of the access to the jaw and does not itself affect the condition of the patient in any negative way. K7 uses a Windows based software program.

It was thus possible to objectively record and represent graphically the kinetic movement of the jaw pre- and post-treatment, and to measure the differences. The results were then assessed statistically; all average differences in excess of 5 percent were deemed to be significant.

In the first session, the jaw closing speed of each test subject was measured twice using the K7 measuring system. This was followed by placebo/sham treatment and, once again, the measurements were recorded twice. A second session took place an average of eight weeks later. Here, the same procedure was repeated, with the exception that an actual CsTh treatment was administered.

The metrological working hypothesis of the study - the K7 measuring system

An essential prerequisite for verifying the craniosacral hypothesis is a measuring system. The K7 measuring system is suitable for measuring mandibular opening and closing speeds (velocity) with sufficient accuracy in order to map the effect of craniosacral interventions [12,13]. The K7 works by using an array of sensors, specifically eight magnetometers, (four on each side), which are held in place. The array is worn like a pair of eye glasses on the ears and nose. A very small calibrated and tuned magnet is affixed to the mandibular incisors, and the movement of the jaw/magnet is displayed on a computer screen. This system facilitates the mapping of craniosacral interventions with objective data. The following have been assumed as the metrological working hypothesis of our study:

- $\quad$ The K7 jaw tracking measuring system accurately measures the opening and closing speeds of the mandible as it moves through time and space.

- $\quad$ The K7 jaw tracking measuring system offers the possibility of a kinesiographic measurement of the maximum speed of the mandible through until, and at the point of, terminal tooth contact.

- The printout of such movement includes both a descriptive and numerical analysis of all the speeds of mandibular movements, including the speed of the mandible at the time of terminal tooth contact. 


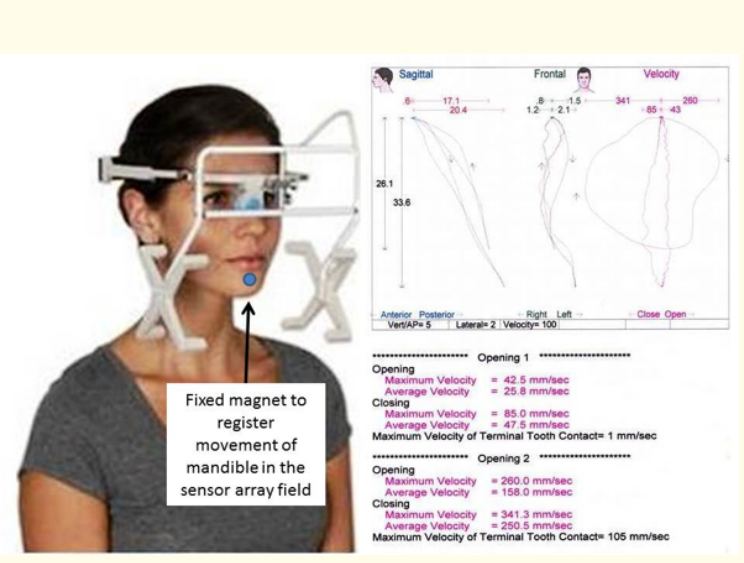

Figure 1: Sensor array (left panel) and printout of analysis of all the speeds of mandibular movements (right panel).

\section{The craniosacral working hypothesis of the study}

Five CsT were used in this study in order to ascertain and quantitatively measure the effect of various osteopathic treatments on jaw function as measured by the speed at the point of terminal tooth contact. The effect of five different CsT on the kinematics of the craniomandibular system have been validated kinematographically with the K7. These are as follows: cranial base release; parietal lift; sphenoid lift; alternate rotation of the temporal bone (i.e., Os temporale); and compression/decompression of the mandible.

- Hypothesis I: Actual treatment is effective in the overall random sample.

- Hypothesis II: Placebo/sham treatment is less effective in the overall random sample than with actual treatment.

- Hypothesis III: Actual treatment is more effective in subjects requiring treatment than in healthy test subjects.

- Hypothesis IV: Placebo/sham therapy is less effective in subjects requiring treatment compared to the actual treatment group.

\section{The test subject collective}

All test subjects were patients at the Clinic for Integrative Dentistry Munich, Germany and had noninvasive craniomandibular TMJ treatment. They were informed about the nature of the study, and their identities were blinded in the study. So test subjects were prevented from knowing which treatment they had received. The present study was executed as a retrospective case control study and waived approval by IMD-Berlin forensic accredited Institute
DIN EN 15189/DIN EN 17025. All patients gave their written informed consent.

The collective comprised eleven female and eleven male test subjects, aged between 20 and 40, who had not reported any subjective problems with their craniomandibular system (i.e., jaw joints, muscles and teeth). The average age of the female test subjects was 29.9, and for the male test subjects the average was 31.0. The average age of the overall random sample was 30.5. Sub-groups consisted of 17 test subjects not requiring treatment and five test subjects requiring treatment.

Determination of the effectiveness of craniosacral techniques

It was assumed that positive or negative changes to the measured values were the result of the method used in the treatment group.

The difficulty of precluding the effect of the placebo/sham treatment on the results, compared to those for the actual treatment, was counteracted by the fact that placebo/sham treatments were always carried out before the actual treatment. The aim of this was to minimize any subjective effects that could not be eliminated with manual therapy in the post-actual therapy follow-up.

The null hypothesis comprises the statement that the five CsT applied do not produce any statistically relevant changes in terms of the measuring parameters under examination. The effectiveness of the CsT were determined based on the following parameters and measured values (Terminal tooth contact (TT) after slow closing (terminal tooth contact slow(TTs) and terminal tooth contact after fast closing (terminal tooth contact fast (TTf)) before and after actual treatment, and before and after placebo/sham treatment:

If the occlusion is perfect, the final TT speed will be relatively fast. If there is disturbingly early tooth contact, as a noxious influence there is a proprioceptive braking/restraining of the speed of contact between the teeth, and the maximum TT speed will be reduced. The velocity at the time of terminal tooth contact is an assessment criterion for the absence of occlusion disturbance, i.e., the faster the speed at tooth contact, the less disturbance there is during proprioception on tooth contact. Terminal tooth contact thus emerges as an extremely important assessment criterion for the quality of occlusion or, in other words, the integrity of the stomatognathic system. 
Opening and closing speed (velocity in $\mathrm{mm} / \mathrm{sec}$ )

The opening and closing speed were measured twice in an initial session with the $\mathrm{K} 7$ for all test subjects, after which they were treated with the placebo/sham therapy, and then measured twice again. The measurement reports were evaluated statistically in average:

- $\quad$ Fast opening speed (op f); average fast opening speed (op af); slow opening speed (op sl); average slow opening speed (op asl) (velocity in $\mathrm{mm} / \mathrm{sec}$ )

- $\quad$ Slow closing speed (cl sl); average slow closing speed (cl asl); fast closing speed (cl f); average fast closing speed (cl af) (velocity in $\mathrm{mm} / \mathrm{sec}$ )

\section{Statistical methods}

Statistical analysis was performed using IBM SPSS, version 19 (IBM Corporation, Armonk, NY, USA). All data was presented as a mean \pm standard mean error. The two-sided unpaired t-test was used in determining the differences in the groups while a Spearman coefficient was used in examining the correlations' presence. Data was considered significant where the value was $\mathrm{p} \leq .002$.

\section{Results}

The initial functional diagnostics performed with the K7 showed normal findings, not requiring treatment, in three female and nine male test subjects, and kinesiographic findings requiring treatment in three female and two male test subjects. The threshold indication for treatment was defined as severe lateral deviation in the opening movement of the mandible from a closed jaw (teeth together) position. Among those healthy test subjects with no lateral deviation, but with severely reduced terminal tooth contact due to mechanical occlusal problems, were eight test subjects with occlusal disturbances and nine without occlusal disturbances. The measurement results from this study are divided into three sections:

- $\quad$ The measured values for the overall random sample before and after actual treatment (to verify hypothesis I).

- $\quad$ The measured values for test subjects requiring treatment (to verify hypotheses III).

- $\quad$ The measured values for test subjects requiring treatment (to verify hypotheses IV).
The color codes used in the graphs are as follows

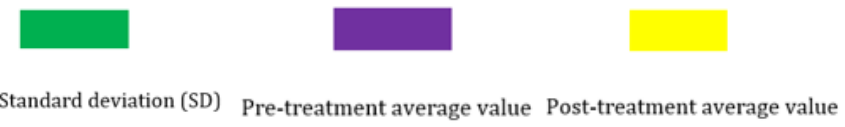

Figure

Comparison of pre- and post-actual treatment measured values - hypothesis I

Figure 2 shows the maximum fast (Vmax cl f) and average fast (average cl f) closing speeds before and after actual treatment. The maximum fast closing speed increased by $14.5 \%$ after treatment. This increase is significant $(\mathrm{P} \leq .002)$. The increase in the average closing speeds was $8.5 \%$ and is not significant $(\mathrm{P} \leq .102)$.

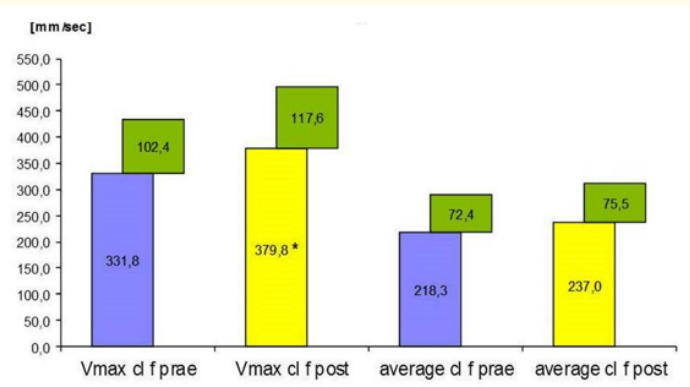

Figure 2: Fast closing speeds (cl f) pre- and post-actual (CsT) treatment.

CsT/placebo: comparison $(n=22)$ of fast closing speeds (cl f) pre- and post-treatment - hypothesis II

Figure 3 shows that fast closing speeds increase significantly following actual treatment compared to placebo/sham treatment:

- $\quad c l$ f increased significantly after actual treatment by 48 $\mathrm{mm} / \mathrm{sec} ; \mathrm{p} \leq .002$.

- $\quad \mathrm{cl}$ f decreased significantly after placebo treatment by 8.6 $\mathrm{mm} / \mathrm{sec} ; \mathrm{p} \leq .049$.

- $\quad$ cl f was $5.7 \mathrm{~mm} / \mathrm{sec}$ faster after actual treatment than before placebo treatment; $\mathrm{p} \leq .779$.

- $\quad c l$ f was $33.3 \mathrm{~mm} / \mathrm{sec}$ faster after placebo treatment than before actual treatment; $\mathrm{p} \leq .365$. 


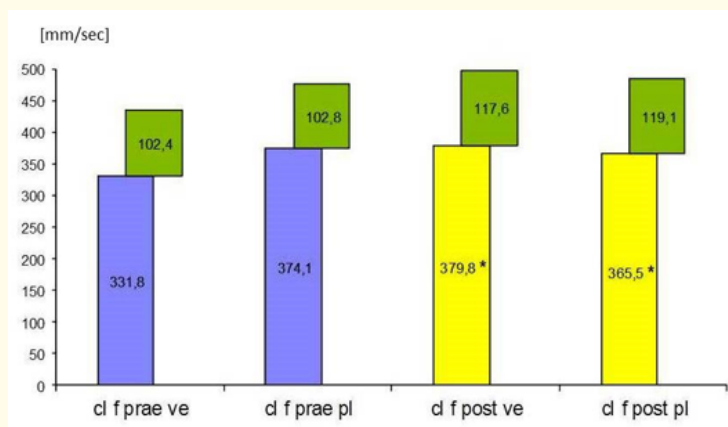

Figure 3: CsT/placebo comparison of fast closing speeds (cl f) before and after treatment

The standard deviations were as follows: 30.6 for cl f pre-CsT, $27.5 \%$ for $\mathrm{l} f$ pre-pl, $31 \%$ for cl f post-CsT and $32.6 \%$ for cl f post-pl of the respective average values.

CsT/placebo: Comparison of fast opening speeds (op f) preand post- treatment

A comparison of fast opening speed measurements pre- and post-placebo treatment showed a significant decrease of $16.6 \mathrm{~mm} /$ sec $(-6 \%)$. A significant change in measured values pre- and postplacebo treatment was found $(\mathrm{p} \leq 0.034)$; the fast opening speeds fell by $18.9 \mathrm{~mm} / \mathrm{sec}(6.8 \%)$ after placebo treatment. There was a difference of $0.9 \mathrm{~mm} / \mathrm{sec}(\mathrm{p} \leq 0.291)$ between post-CsT (op f postCsT) and post-placebo treatment (op f post-pl).

Figure 4 also shows that fast opening speeds reduced significantly following placebo treatment:

- op f increased by $3.3 \mathrm{~mm} / \mathrm{sec}$ after CsT treatment; $\mathrm{p} \leq .985$.

- op f was $4.5 \mathrm{~mm} / \mathrm{sec}$ faster after actual treatment than before placebo treatment; $\mathrm{p} \leq .895$.

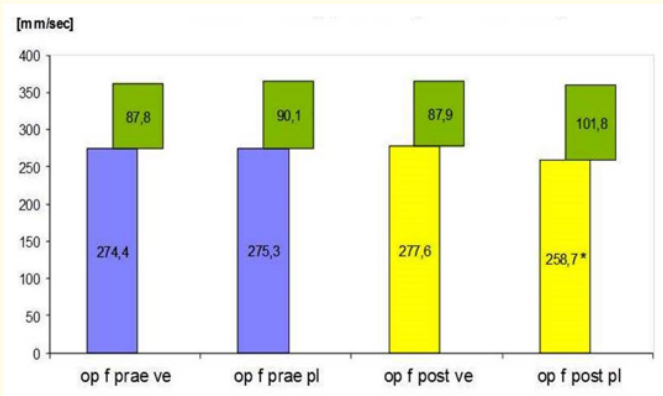

Figure 4: CsT/placebo comparison of fast opening speeds (op f) before and after treatment.
The standard deviations were as follows: $32 \%$ for op f pre-CsT, $32.7 \%$ for op f pre-pl, $31.7 \%$ for op f post-CsT and $39.4 \%$ for op $\mathrm{f}$ post-pl of the respective average values.

CsT/placebo: Comparison of terminal tooth contact, slow (TT sl) and fast (TT f), pre- and post-treatment in test subjects without occlusion problems $(n=9)$

Figure 5 charts the CsT/placebo comparison of TT sl and TT f values before and after treatment in nine healthy test subjects without occlusion problems. The key point is the significant increase in TT f from 48.72 to $75.8 \mathrm{~mm} / \mathrm{sec}$ ( $\mathrm{p} \leq 0.008$ ) after CsT treatment. This represents an increase of $55.8 \%$. The standard deviations were $79.4 \%$ for TT f pre-CsT and $68.4 \%$ for TT f post-CsT.

- TT sl showed an insignificant decrease from 8.4 to $7.4 \mathrm{~mm} /$ sec after CsT treatment

- $\quad$ TT sl showed an insignificant decrease from 7.7 to $7.6 \mathrm{~mm} /$ sec after placebo treatment.

- $\quad$ TT $\mathrm{f}$ showed an insignificant decrease of $1.57 \mathrm{~mm} / \mathrm{sec}$ after placebo treatment.

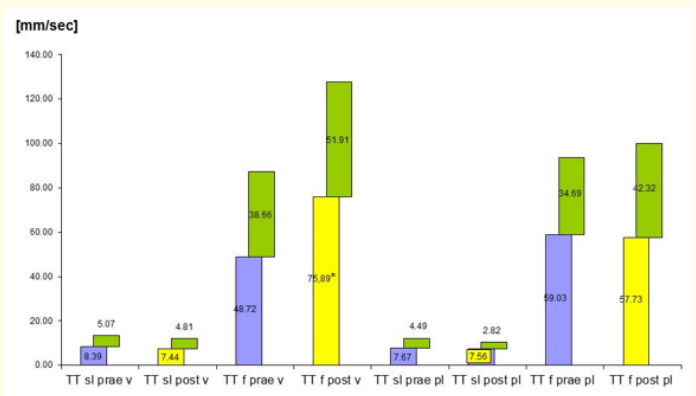

Figure 5: CsT/placebo comparison of terminal tooth contact, slow (TTsl) and fast (TT f), before and after treatment in test subjects without occlusion problems $(n=9)$.

CsT/placebo: Comparison of opening speeds, slow (op s) and fast (op f), pre- and post- treatment, in test subjects requiring treatment $(n=5)$

The respective value pairs are not significant.

The individual changes in measured values show the following:

- op sl decreased by $9.1 \mathrm{~mm} / \mathrm{sec}$ after CsT treatment; $\mathrm{p} \leq 0.313$.

- $\quad$ op sl increased by $8.1 \mathrm{~mm} / \mathrm{sec}$ after placebo/sham treatment; $\mathrm{p} \leq 0.438$.

- $\quad$ op sl post-CsT was $2.9 \mathrm{~mm} / \mathrm{sec}$ faster than op sl pre-pl, and at $\mathrm{p} \leq 0.999$ is not significantly different. 
The standard deviations were as follows: $8.8 \%$ for op sl pre-CsT, $23 . \%$ for op sl pre-pl, $34 \%$ for op sl post-CsT and $26.7 \%$ for op sl post-pl of the respective average values.

- op f decreased by $8.9 \mathrm{~mm} / \mathrm{sec}$ after CsT treatment; $\mathrm{p} \leq 0.999$.

- $\quad$ op f decreased by $9.6 \mathrm{~mm} / \mathrm{sec}$ after placebo/sham treatment; $\mathrm{p} \leq 0.813$.

- $\quad$ op f post-CsT is not significantly different to op f pre-pl; $\mathrm{p} \leq 0.625$.

The standard deviations were as follows: $24.6 \%$ for op $\mathrm{f}$ preCsT, $29.8 \%$ for op f pre-pl, $28.7 \%$ for op f post-CsT and $35.5 \%$ op f post-pl of the respective average values.

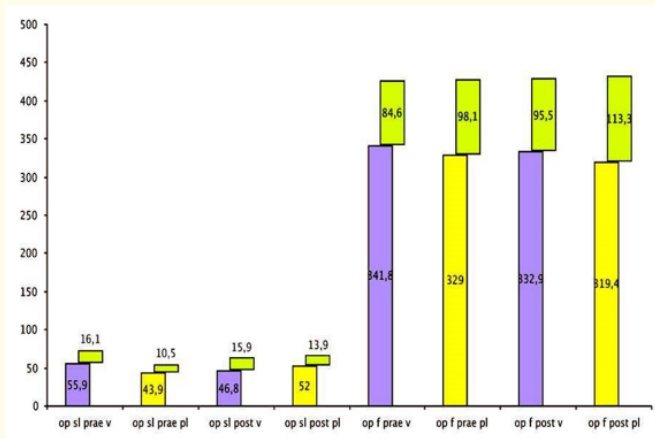

Figure 6: CsT/placebo comparison of opening speeds, slow (op sl) and fast (op f), pre- and post-treatment in test subjects requiring treatment.

Closing speeds in the group of test subjects requiring treatment $(n=5)$ before and after treatment, comparing CsT and placebo treatment

The individual changes in measured values show the following:

- cl sl decreased by $1.0 \mathrm{~mm} / \mathrm{sec}$ after CsT treatment; $\mathrm{p} \leq 0.813$.

- $\quad$ cl sl increased by $24.4 \mathrm{~mm} / \mathrm{sec}$ after placebo treatment; $\mathrm{p} \leq 0.313$.

- $\quad$ cl sl post-CsT was $237 \mathrm{~mm} / \mathrm{sec}$ faster than cl sl pre-pl, but at $\leq 0.188$ not significantly different.

The standard deviations were as follows: $38.5 \%$ for cl sl preCsT, $21.8 \%$ for cl sl pre-pl, $50.1 \%$ for cl sl post-CsT and $53.5 \%$ for $\mathrm{cl}$ sl post-pl of the respective average values.
- $\quad \mathrm{cl} \mathrm{f}$ decreased by $23 \mathrm{~mm} / \mathrm{sec}$ after placebo treatment; $\mathrm{p} \leq 0.438$

- $\quad \mathrm{cl} \mathrm{f}$ post-CsT is not significantly different to cl f pre-pl; $\mathrm{p} \leq 313$.

The standard deviations were as follows: $32 \%$ for cl f pre-CsT, $30.5 \%$ for cl f pre-pl, $30.8 \%$ for cl f post-CsT and $37.2 \%$ for cl f post$\mathrm{pl}$ of the respective average values.

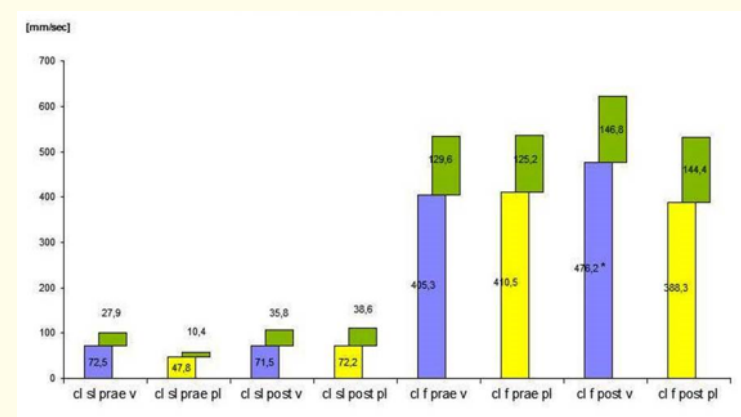

Figure 7: CsT/placebo comparison of closing speeds, slow (op sl) and fast (op f), pre- and post-treatment in test subjects requiring treatment.

CsT/placebo: Comparison of terminal tooth contact, slow (TT sl) and fast (TT f), pre- and post-treatment in test subjects requiring treatment $(\mathrm{n}=5)$

The results relating to hypothesis III (i.e., CsT treatment is effective in subjects requiring treatment) and IV (i.e., in this random sample, placebo treatment is less effective in subjects requiring treatment compared to actual treatment) are shown in Figure 8. With respect to the validity of hypothesis I, the chart also shows a significant rise in fast closing speed after CsT treatment in a comparison of the values pre- and post-treatment.

The terminal tooth contact on slow closing before CsT treatment (TT sl pre-CsT), on slow closing before placebo treatment (TT sl pre-pl), on slow closing after CsT treatment (TT sl post-CsT), on slow closing after placebo treatment (TT sl post-pl), on fast closing before CsT treatment (TT f pre-CsT), on fast closing before placebo treatment (TT f pre-pl), on fast closing after CsT treatment (TT f post-CsT) and on fast closing after placebo treatment (TT f post-pl) are shown in Figure 8. 


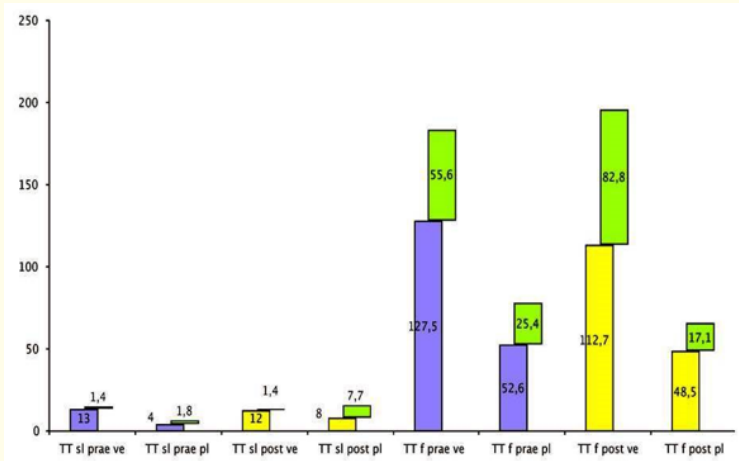

Figure 8: CsT/placebo comparison of terminal tooth contact, slow (TTsl) and fast (TT f), before and after treatment in test subjects requiring treatment $(n=5)$.

The changes in the measured values show the following:

- $\quad$ TT sl decreased by $1.0 \mathrm{~mm} / \mathrm{sec}$ after CsT treatment; $\mathrm{p} \leq 0.625$.

- $\quad$ TT sl increased by $4.0 \mathrm{~mm} / \mathrm{sec}$ after placebo treatment; $\mathrm{p} \leq 0.188$.

- $\quad$ TT sl post-CsT is not significantly different to TT sl pre-pl; $\mathrm{p} \leq 0.125$.

The standard deviations were as follows: $10.7 \%$ for TT sl preCsT, $45 \%$ for TT sl pre-pl, $11.7 \%$ for TT sl post-CsT and $96.3 \%$ for TT sl post-pl of the respective average values.

- $\quad$ TT f decreased $15.2 \mathrm{~mm} / \mathrm{sec}$ after CsT treatment; $\leq 0.999$.

- $\quad$ TT f decreased by $4.1 \mathrm{~mm} / \mathrm{sec}$ after placebo treatment; $\mathrm{p} \leq 0.999$.

- $\quad$ TT f post-CsT is not significantly different to TT f pre-pl; $\mathrm{p} \leq 0.81$.

The standard deviations were as follows:43.6\% for TT f pre-CsT, $48.3 \%$ for TT f pre-pl, $73.5 \%$ for TT f post-CsT and $35.3 \%$ for TT $\mathrm{f}$ post-pl of the respective average values.

\section{Summary of results}

Following placebo/sham treatment, a significant increase in the mandible closing speed and a decrease in the opening speed were recorded for the overall random sample. The mandible closing speed was clearly faster after CsTh treatment in both test subjects requiring treatment and healthy test subjects. A significant decrease in jaw opening speed was recorded in healthy test subjects after placebo/sham treatment. Terminal tooth contact during fast jaw closure was significantly faster in both healthy test subjects and those test subjects requiring treatment with no occlusion difficulties, in both treatment groups, i.e., after CsTh or placebo treatment (Figure 9).

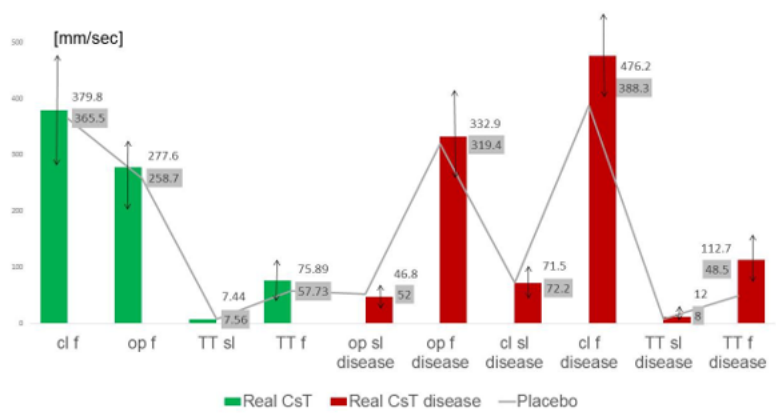

Figure 9: Summary of results. Legend: Green columns show cohort without occlusal or TMJ problems ; red columns show cohort with occlusal and TMJ problems; grey line shows placebo.

The acceptance level among test subjects was extremely high. There were no test subjects for whom the method of taking measurements was cause for withdrawal from the study.

\section{Discussion}

The measured values from the study are significant for the following reasons:

- $\quad$ Fast closing speeds were faster after CsTh treatment compared to placebo/sham treatment, and increased significantly after CsTh treatment of both healthy test subjects and those subjects requiring treatment.

- $\quad$ Fast opening speeds decreased significantly after placebo treatment.

- $\quad$ TT increased for fast closing in both healthy test subjects without occlusion problems, and test subjects requiring treatment without occlusion problems.

The procedures required to undertake this study were repeated with a high degree of consistency. Using the data, it was possible to demonstrate that movements of the jaw joint apparatus, which 
may be measured with the K7, may be influenced with the CsT used here.

\section{Verification of hypotheses}

Hypothesis I: assumed that CsT would be effective in the overall random sample when the pre- and post-treatment values were compared. The validity of this assumption can be confirmed with respect to the maximum closing speeds. A comparison of pre- and post-treatment values shows a significant increase in the fast closing speed of $48 \mathrm{~mm} / \mathrm{sec}(14.5 \%$; $\leq 0.0002)$.

Hypothesis II: concerns the maximum fast closing speeds and fast opening speeds obtained in a comparison of CsT and placebo treatment. Both speeds increased significantly, i.e., by $48 \mathrm{~mm} / \mathrm{sec}$ (14.5\%; $\mathrm{p} \leq 0.002)$ and $16.6 \mathrm{~mm} / \mathrm{sec}(6 \% ; \mathrm{p} \leq 0.034)$ respectively after CsT. At the same time, the fast closing speeds decreased significantly, i.e., by $8.6 \mathrm{~mm} / \mathrm{sec}(=2.3 \%$; $\leq 0049)$ after placebo treatment. These measuring parameters demonstrate that placebo treatment is less effective than CsT and, furthermore, appear to confirm that CsT produce the anticipated changes. This statistically relevant increase in fast closing speeds indicates the following:

- The CsT applied here affect the closing speeds of the craniomandibular system and the associated contiguous ligamentous structures.

- $\quad$ CsT specifically affect the closing speeds with respect to any increase in speed.

- This CsTh effect is physiologically beneficial as it may be assumed that the craniomandibular apparatus is in a more optimal functional state in terms of the muscles, capsular and ligament apparatus and its proprioception, with reduced strain/restriction, the faster it is able to move.

The significant decrease in fast closing speeds after placebo treatment may be explained by the excessive opening movements required of test subjects as part of the process of recording the kinematics of the jaw joint. These opening movements cause fatigue in the jaw joint apparatus in the absence of CsT, as a result of the severe expansion processes in the ligaments and muscular parts of the jaw joint. The reduction in fast opening speeds after placebo treatment also confirms hypothesis II. The results support the argument that in the CsT group this fatigue is compensated for by the application of CsT, in contrast to the placebo/sham group where there is no such compensation.
Verification of hypothesis in the group of test subjects requiring treatment

It was determined that a test subject required treatment where the arthrokinematics of the jaw joint were found to be disturbed according to the results of the initial $\mathrm{K} 7$ findings. These functional disturbances were, in principle, assessed as additional load factors for the structures of the jaw joint. The positive effect of the CsT, however, was greater in the random sample than in healthy subjects. This provides an initial indication that the CsT applied here have a positive therapeutic effect on the kinesiographic symptoms. In relation to hypothesis III, CsT for subjects requiring treatment appears to be effective only with respect to fast closing speeds. The $17.5 \%$ (70.9 mm/sec; $\mathrm{p} \leq 0.046)$ increase in these speeds is significantly higher for CsT than placebo/sham treatment when the preand post- treatment values are compared. The drop in fast closing speeds of $18.6 \mathrm{~mm} / \mathrm{sec}(7.2 \%$; $\mathrm{p} \leq 0039)$ after placebo treatment for healthy test subjects and $23 \mathrm{~mm} / \mathrm{sec}(\mathrm{p} \leq 0.438)$ in subjects requiring treatment also indicate poorer compensation for jaw expansion processes and fatigue effects in the absence of effective CsT.

\section{Terminal tooth contact}

The results for TT are also of interest. Above and beyond the research hypotheses, enlightening results were obtained from test subjects without occlusion problems. TT was used primarily to distinguish between occlusion disturbances caused by misaligned teeth or raised crowns and fillings, and problems with the functional structures of the jaw joint, caused by trauma, mandibular dysfunction or high cervical blockages. The significance of CsT for changes in measured TT values can, therefore, only be given due consideration if occlusion disturbances are precluded. Figure 5 illustrates the results of TT for test subjects without occlusion problems, comparing both pre- and post-treatment values, and CsT and placebo/sham treatment. The random sample of test subjects requiring treatment showed an increase in TT from 90.5 to 1325 $\mathrm{mm} / \mathrm{sec}$ after CsT treatment (Figure 8). This represents an increase of $46.4 \%$. A calculation of the significance was not performed due to the fact that the results were from a small random-sample. The standard deviation for this sample was $79.3 \%$ for TT f pre-CsT and 73.7\% for TT f post CsT.

Larger numbers of test subjects would be required in order to verify, firstly, the standard deviations and, secondly, the validity of the considerable increases in measured TT f values. The casuistic 
assessment of these findings seems to be particularly significant because the TT $\mathrm{f}$ increase is high at $46.4 \%$, whereas the placebo produced no discernable change in the measured values. It is possible that the effectiveness of CsTh could be conclusively demonstrated using larger random samples with reference only to the TT f changes.

\section{Study boundaries}

This study was an initial pilot study with a small random sample using the five CsT applied here. As the random samples in this study were small, the inter individual measured value ranges may mask the possible effects of craniosacral osteopathic treatment.

\section{Conclusions}

The data shows that evaluable movements of the mandible, as influenced by the temporomandibular joint (TMJ) and associated musculature, may be documented with values measured using the $\mathrm{K} 7$, and that mandibular movement (synergy, synchronicity, and fluidity) in healthy test subjects may be positively influenced with CsT. Craniomandibular dysfunctions caused by occlusion cannot be improved solely with CsT, and in the case of patients requiring occlusal treatment, CsT alone are not the solution. Arthrokinematic disturbances of the jaw joints can be treated before problems are noticed and, by so doing, serious secondary consequences may be avoided as far as possible. Craniomandibular dysfunctions that are not caused by occlusion may be improved by using the CsT verified here. Most significantly however, these results highlight that CsT may not be regarded as a placebo treatment. The complete elucidation of complex interactions underlying these processes requires further research.

\section{Disclosure Statement}

There are no conflicts of interest.

\section{Bibliography}

1. Upledger JE and Vredevoogd JD. “Lehrbuch der Craniosacralen Therapie I Stuttgart, Karl F Haug Verlag (2003).

2. Sutherland WG. "Teaching in the science of osteopathy, Clarkston, Sutherland Cranial Teaching Foundation (1991): 28.

3. Rocabado M., et al. "Physical therapy and dentistry: an overview". Journal of Craniomandibular Practice 1.1 (1983): 46-49.

4. Gelb J and Gelb M. "Taking the mystique out of the diagnosis and treatment of craniomandibular (TMJ) disorders". International Dental Journal 39 (1998): 129-139.

5. Gresham H and Smithells PA. "Cervical and mandibular posture". Dent Recreation 74 (1954): 261-264.
6. Fink M., et al. "Kraniomandibuläres System und WirbelsäuleFunktionelle Zusammenhänge mit der Zervikal und LendenBecken-Hüft-Region". Man Med 41 (2003): 476-480.

7. Festa F., et al. "Effects of horizontal oscillation of the mandible on the spinal column of the rat in vivo using radiogrphic monitoring". Orthognatodonzia Ital 6 (1997): 539-550.

8. King Hollis H. "Osteopathy in the cranial field: Uncovering challenges and potential applications". The Journal of the American Osteopathic Association 102.7 (2002): 367-369.

9. King HH. "Manual Craniosacral Therapy May Reduce Symptoms of Migraine Headache". The Journal of the American Osteopathic Association 117.1 (2017): 59.

10. Edward E. "Craniosacral therapy:A systematic review of the clinical evidence". Focus on Alternative and Complimentary Therapies 17.4 (2012): 197-201.

11. Hartman SE and Norton JM. "Craniosacral therapy is not medicine”. Physical Therapy 83.11 (2002): 1146-1147.

12. Moran RW and Gibbons P. "Interexaminer and Intraexaminer Reliability of the Cranial Rhythmic Impulse at the Head and Sacrum". Journal of Manipulative and Physiological Therapeutics 24.3 (2001): 183-190.

13. Schöttl W. "Die cranio-mandibuläre-Regulation. Heidelberg, Hüthig Verlag (1991):106.

14. Rang NG and Höppner S. "SCO Cranio Sacral Osteopathie, Stuttgart, Hippokrates Verlag (1997).

15. Jankelson RR. "Analysis of maximal electromyographic activity of the masseter and anterior temporalis muscles in myocentric and habitual centric in temporomandibular joint and musculoskeletal dysfunction. Pathophysiology of Head and Neck Musculoskeletal Disorders. International College of Craniomandibular Orthopedics. Basel, Karger 7 (1990): 83-98.

16. Schöttl R. Geleitwort zu dem Buch von Harry JM von Piekartz: Kiefer Gesichts- und Zervikalregion, Stuttgart, Thieme, Verlag (2005).

\section{Assets from publication with us}

- Prompt Acknowledgement after receiving the article

- Thorough Double blinded peer review

- Rapid Publication

- Issue of Publication Certificate

- High visibility of your Published work

Website: www.actascientific.com/

Submit Article: www.actascientific.com/submission.php Email us: editor@actascientific.com Contact us: +919182824667 